DOl:

REVISION

\title{
Infarto renal: revisión sistemática sobre síntomas de sospecha clínica y repercusión sobre función renal
}

Ana Carina Pizzarossa

ORCID: 0000-0001-5449-9124 Internista. Asistente Clínica Médica.

UdelaR.

Marina Rodríguez ORCID: 0000-0001-6452-7897 Internista. Asistente Clínica Médica.

UdelaR.

\section{Renal infarction: systematic review of symptoms of clinical suspicion and repercussion on renal function}

Infarto renal: revisão sistemática dos sintomas de suspeita clínica e repercussão na função renal

Resumen: El infarto renal es una entidad poco frecuente y está sub-diagnosticada por lo que para su diagnóstico se necesita un alto índice de sospecha clínica. Objetivo: Determinar las características clínicas y paraclínicas más frecuentes en el infarto renal, destacando la repercusión en la función renal. Metodología: Se realizó una revisión sistemática de serie de casos en PUBMED y SciELO con los términos: "renal infarction" y "renal infarct". Se estudiaron variables: demográficas, clínicas y paraclínicas. En el caso de las variables paraclínicas se analizó el porcentaje de pacientes con valores elevados de cada una de las mismas. El análisis estadístico fue realizado con EPI INFO 7. 2. Resultados: Los síntomas y signos más frecuentes fueron oliguria, dolor en flanco y abdominal, nauseas, fiebre y vómitos. Los parámetros paraclínicos alterados fueron: LDH, PCR y glóbulos blancos. Se constató injuria renal aguda en $30.8 \%$ de los pacientes, la progresión a enfermedad renal crónica se observó en 17.3\% y la progresión a enfermedad renal terminal se observó en $5.2 \%$ de los pacientes. Discusión y conclusiones: La determinación de las características clínicas y paraclínicas más frecuentes del infarto renal, se pueden utilizar para disminuir el retraso diagnóstico, lo que tiene consecuencias terapéuticas.

Palabras clave: Infarto renal, dolor en flanco, LDH, injuria renal.

Abstract: Renal infarction is a rare entity and is under-diagnosed, so a high index of clinical suspicion is needed for its diagnosis. Objective: To determine the most frequent clinical and paraclinical characteristics in renal infarction, highlighting the impact on renal function. Methodology: A systematic review of a series of cases was carried out in PUBMED and SciELO with the terms: "renal infarction" and "renal infarct". Variables were studied: demographic, clinical and paraclinical. In the case of paraclinical variables, the percentage of patients with high values of each of them was analyzed. The statistical analysis was performed with EPI INFO 7. 2. Results: The most frequent symptoms and signs were oliguria, flank and abdominal pain, nausea, fever and vomiting. Paraclinical parameters altered were: LDH, CRP and white blood cells. Acute renal injury was found in $30.8 \%$ of patients, progression to chronic kidney disease was observed in $17.3 \%$ and progression to end-stage renal disease was observed in $5.2 \%$ of patients. Discussion and conclusions: The determination of the most frequent clinical and paraclinical characteristics of renal infarction can be used to reduce the diagnostic delay, which has therapeutic consequences.

Key words: Renal infarction, flank pain, LDH, renal injury.

Resumo: O infarto renal é uma entidade rara e é subdiagnosticada, portanto, um alto índice de suspeita clínica é necessário para o seu diagnóstico. Objetivo: Determinar as características clínicas e paraclínicas mais frequentes no infarto renal, destacando o impacto na função renal. Metodologia: Uma revisão sistemática de uma série de casos foi realizada em PUBMED e SciELO com os termos: "infarto renal" e "infarto renal". As variáveis foram estudadas: demográficas, 
clínicas e paraclinicais. No caso de variáveis paraclínicas, foi analisada a porcentagem de pacientes com valores altos de cada um deles. A análise estatística foi realizada com o EPI INFO 7. 2. Resultados: Os sintomas e sinais mais frequentes foram oligúria, dor no flanco e abdominal, náusea, febre e vômitos. Parâmetros clínicos alterados foram: LDH, PCR e glóbulos brancos. A lesão renal aguda foi encontrada em $30,8 \%$ dos pacientes, a progressão para doença renal crônica foi observada em $17,3 \%$ e a progressão para doença renal terminal em $5,2 \%$ dos pacientes. Discussão e conclusões: A determinação das características clínicas e paraclínicas mais frequentes do infarto renal pode ser utilizada para reduzir o retardo no diagnóstico, o que tem consequências terapêuticas.

Palavras-chave: Infarto renal, dor no flanco, LDH, lesão renal.

Recibido: 20/03/2019 Aceptado: 14/05/2019

Clínica Médica "C". Hospital de Clínicas “Dr. Manuel Quintela". Facultad de Medicina. Universidad de la República (UdelaR) Montevideo, Uruguay.

Correspondencia: E-mail: carina491986@gmail.com 


\section{Introducción}

El infarto renal es una entidad poco frecuente, sub-diagnosticada, de baja incidencia y la información de esta patología se sustenta en reporte de casos ${ }^{(1,2)}$. Una de las causas de este sub-diagnóstico y/o retraso diagnóstico es la variedad de diagnósticos diferenciales que se plantean frente a un dolor unilateral en flanco. Entre ellos se citan: cólico nefrítico, pielonefritis, cuadro abdominal agudo y menos frecuentemente síndrome coronario agudo. Por eso la sospecha diagnostica es vital para un diagnóstico oportuno dado que esto condiciona el arsenal terapéutico y eventualmente el impacto en la función renal. ${ }^{(3,4)}$

Los objetivos de esta revisión son:

1) determinar las características clínicas y paraclínicas más frecuentes en el infarto renal y

2) estudiar la repercusión en la función renal

\section{Metodología}

Se realizó una búsqueda bibliográfica incluyendo las siguientes bases de datos: Medline (interfase Pubmed), SciElo de acuerdo a las siguientes palabras claves (MESH term): renal AND infarction, infarto AND renal.

\section{Criterios de inclusión:}

1) redactadas en inglés o español,

2) con pacientes mayores de 14 años,

3) inclusión de 5 o más pacientes,

4) serie que incluyan infartos renales de más de una etiología,

5) presencia de estudio de características clínicas y paraclínicas,

6) diagnostico antemorten,

7) diagnostico con tomografía computada, centellograma de perfusión renal, resonancia nuclear magnética y angiografía.

\section{Criterios de exclusión:}

1) diagnostico postmorten,

2) diagnostico con otros métodos no citados en punto 7 de criterios de inclusión.

3) series que incluyeran infartos renales de una sola etiología.

Las variables estudiadas fueron: demográficas (edad, sexo), clínicas (presencia de dolor abdominal, dolor en flanco, nauseas, vómitos, fiebre, confusión, disnea, diarrea, dolor torácico), paraclínicas (elevación de los siguientes parámetros: glóbulos blancos, AST, ALT, LDH, PCR, presencia de hematuria y proteinuria), impacto en función renal (insuficiencia renal aguda, progresión a enfermedad renal crónica, progresión a enfermedad renal en etapa terminal).

La injuria renal aguda se definió según los parámetros de la clasificación RIFLE, AKIN o KDIGO, o en su defecto por disminución del filtrado glomerular o aumento de la creatinina sin cumplir todos los criterios de las clasificaciones nombradas. ${ }^{(5-7)}$

a) RIFLE: aumento en creatinina sérica de 100 a $199 \%$ o una diuresis menor a $0.5 \mathrm{ml} / \mathrm{kg} /$ hora por 12 a 24 horas.

b) AKIN: aumento de creatinina mayor o igual a $0.3 \mathrm{mg} / \mathrm{dl}$ o aumento mayor o igual al $50 \%$ dentro de 48 horas o diuresis menor a $0.5 \mathrm{ml} / \mathrm{kg} / \mathrm{hora}$ por más de 6 horas.

c) KDIGO: aumento en creatinina sérica mayor o igual a $0.3 \mathrm{mg} / \mathrm{dl}$ dentro de 48 horas o un aumento mayor o igual al $50 \%$ dentro de 7 días o una diuresis menor a $0.5 \mathrm{ml} / \mathrm{kg} / \mathrm{hora}$ por más de 6 horas.

Se definió a la enfermedad renal crónica basado en la presencia de daño renal (anormalidades estructurales o funcionales además de un filtrado glomerular alterado) o disminución de la función renal (tasa de filtración glomerular menor a $60 \mathrm{ml} / \mathrm{min} / 1.73 \mathrm{~m} 2$ ) por 3 meses o más, sin importar la causa.

O en su defecto al aumento persistente de la creatininemia por encima de los valores establecidos por el hospital. 
Se definió a la enfermedad renal terminal como aquella que necesita tratamiento de reemplazo de función renal por más de 3 meses.

Las variables categóricas fueron expresadas en porcentaje y las continuas como medias con desvío estándar o medianas con rango intercuartílico según lo expresado en los artículos revisados. En el caso de las variables paraclínicas se analizó el porcentaje de pacientes con valores elevados de cada una de las mismas. El análisis estadístico fue realizado con EPI INFO 7. 2.

\section{Resultados}

\section{A) Selección artícullos}

De los 76683 artículos que arrojo esta búsqueda (76538 en Medline y 145 en SciElo) realizada en los días 23-27 de octubre de 2017, se procedió a filtrar los artículos por el título, idioma y valorando si incluían más de 5 casos. De esta forma se seleccionaron 42 artículos, cuyos abstracts fueron estudiados por 2 revisores independientes aplicando los criterios planteados anteriormente. Se descartaron 3 artículos por tener solo diagnósticos postmorten o por diagnósticos solo basados en datos clínicos y pielografía, 3 por solo incluir infartos renales de una sola etiología, 11 porque no analizaban características clínicas y paraclínicas y 1 porque había una publicación posterior del mismo hospital que abarcaba los mismos casos. ${ }^{(1,2,9-24)}$

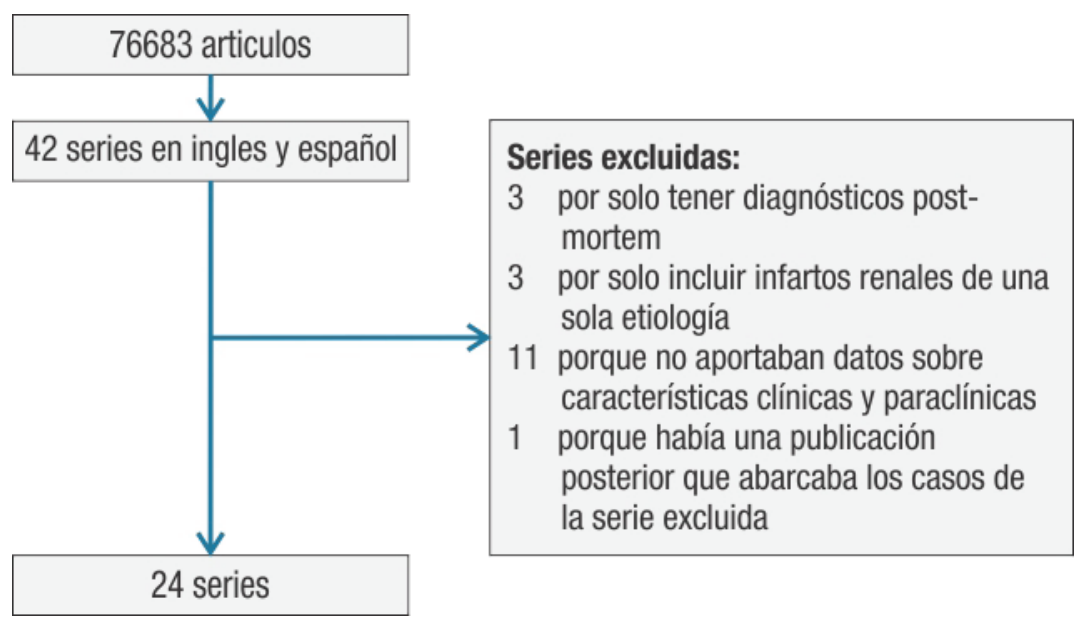

Finalmente, 24 artículos cumplieron los requisitos, que incluyen 1330 pacientes. 22 eran retrospectivas y 2 eran prospectivos (Bolderman y Rhee) ${ }^{(25-26)}$. Se destaca que no hay series de casos nacionales.

En la tabla 1 se presentan criterios de exclusión adicionales a los ya propuestos que tienen algunas de las series.

\begin{tabular}{|l|l|}
\multicolumn{2}{c|}{ Criterios de exclusión de pacientes adicionales } \\
\hline Antopolsky & Se excluye infarto renal de causa traumática y post-operatorio \\
\hline Bolderman & Se excluye infarto renal por shock, iatrogénico y por trauma \\
\hline Bourgalt & $\begin{array}{l}\text { Se excluye infarto renal post-trasplante, post cirugía vascular y angioplastia } \\
\text { percutánea o transluminal }\end{array}$ \\
\hline Caravaca-Fontán & Se excluye infarto renal en trasplantados \\
\hline Huang & Se excluye infarto renal de causa traumática y de menores de 14 años \\
\hline Kwon & $\begin{array}{l}\text { Se excluye infarto renal en pacientes con enfermedad renal crónica previa y menores } \\
\text { de 20años }\end{array}$ \\
\hline Lessman & $\begin{array}{l}\text { Se excluye infarto renal de causa traumática o post cirugía o por embolia } \\
\text { aterotromboembolica }\end{array}$ \\
\hline Yang & Se excluyen pacientes menores de 18 años \\
\hline
\end{tabular}

En la tabla 2 se presentan los datos más relevantes de los 24 artículos seleccionados incluyendo: tamaño de la muestra, características demográficas de los pacientes, características clínicas y paraclínicas del infarto renal. 


\begin{tabular}{|c|c|c|c|c|}
\hline Autor & Título & Año & Revista & Resultados \\
\hline $\begin{array}{l}\text { Lessman } \\
\text { R, Johnson } \\
\text { S, Courn J, } \\
\text { Kaufman J. }\end{array}$ & $\begin{array}{l}\text { Renal Artery } \\
\text { Embolism. } \\
\text { Clinical } \\
\text { Features and } \\
\text { Long-Term } \\
\text { Follow-up of } 17 \\
\text { Cases. }\end{array}$ & 1978 & $\begin{array}{l}\text { Ann Intern } \\
\text { Med. }\end{array}$ & $\begin{array}{l}17 \text { casos incluídos, } 8 \text { presentaron } \\
\text { fiebre, } 7 \text { dolor en flanco, } 14 \text { tienen } \\
\text { LDH elevada y } 13 \text { presentaron IRA }\end{array}$ \\
\hline $\begin{array}{l}\text { Winzelberg GG, } \\
\text { Hull JD, Agar } \\
\text { JWM, Rose BD, } \\
\text { Pletka PG. }\end{array}$ & $\begin{array}{l}\text { Elevation of } \\
\text { Serum Lactate } \\
\text { Dehydrogenase } \\
\text { Levels in Renal } \\
\text { Infarction. }\end{array}$ & 1979 & JAMA & $\begin{array}{l}5 \text { casos, } 3 \text { presentaron fiebre, } \\
1 \text { dolor en flanco, } 5 \text { tienen LDH } \\
\text { elevada y } 2 \text { presentaron IRA }\end{array}$ \\
\hline $\begin{array}{l}\text { Iga K, Izumi } \\
\text { C, Nakano A, } \\
\text { Sakanoue Y, } \\
\text { Kitaguchi S, } \\
\text { Himura Y et al. }\end{array}$ & $\begin{array}{l}\text { Problems in the } \\
\text { initial diagnosis } \\
\text { of renal } \\
\text { infarction. }\end{array}$ & 1997 & $\begin{array}{l}\text { Internal } \\
\text { medicine } \\
\text { (Tokyo, Japan) }\end{array}$ & $\begin{array}{l}20 \text { casos incluidos, } 12 \text { presentaron } \\
\text { hematuria, } 10 \text { dolor en flanco y/o } \\
\text { abdominal y } 12 \text { presentaron IRA }\end{array}$ \\
\hline $\begin{array}{l}\text { Domanovits } \\
\text { H, Paulis M, } \\
\text { Nikfardjam } \\
\text { M, Meron G, } \\
\text { Kurkciyan I, } \\
\text { Bankier A, et al. }\end{array}$ & $\begin{array}{l}\text { Acute renal } \\
\text { infarction. } \\
\text { Clinical } \\
\text { characteristics } \\
\text { of } 17 \text { patients. }\end{array}$ & 1999 & $\begin{array}{l}\text { Medicine } \\
\text { (Baltimore). }\end{array}$ & $\begin{array}{l}17 \text { casos incluídos, } 11 \text { presentaron } \\
\text { dolor en flanco, } 17 \text { presentaron } \\
\text { LDH elevada y } 6 \text { presentaron IRA }\end{array}$ \\
\hline $\begin{array}{l}\text { Lumerman JH, } \\
\text { Hom D, Eiley D, } \\
\text { Smith AD }\end{array}$ & $\begin{array}{l}\text { Heightened } \\
\text { Suspicion } \\
\text { and Rapid } \\
\text { Evaluation with } \\
\text { CT for Early } \\
\text { Diagnosis of } \\
\text { Partial Renal } \\
\text { Infarction. }\end{array}$ & 1999 & J Endourol & $\begin{array}{l}7 \text { casos incluídos, } 4 \text { presentaron } \\
\text { hematuria y nauseas o vómitos, } 3 \\
\text { dolor en flanco y } 7 \text { LDH elevada }\end{array}$ \\
\hline $\begin{array}{l}\text { Korzets Z, } \\
\text { Plotkin E, } \\
\text { Bernheim J, } \\
\text { Zissin R. } \\
\end{array}$ & $\begin{array}{l}\text { The clinical } \\
\text { spectrum of } \\
\text { acute renal } \\
\text { infarction. }\end{array}$ & 2002 & $\begin{array}{l}\text { Isr Med Assoc } \\
\mathrm{J}\end{array}$ & $\begin{array}{l}11 \text { casos incluídos, } 10 \text { presentaron } \\
\text { dolor en flanco, } 9 \text { LDH elevada y } 2 \\
\text { presentaron IRA }\end{array}$ \\
\hline $\begin{array}{l}\text { Bolderman } \\
\text { R, Oyen R, } \\
\text { Verrijcken A, } \\
\text { Knockaert D, } \\
\text { Vanderschueren } \\
\text { S }\end{array}$ & $\begin{array}{l}\text { Idiopathic renal } \\
\text { infarction }\end{array}$ & 2006 & $\begin{array}{l}\text { American } \\
\text { Journal of } \\
\text { Medicine }\end{array}$ & $\begin{array}{l}27 \text { casos incluídos, } 17 \text { presentaron } \\
\text { náuseas y vómitos y } 16 \\
\text { presentaron dolor de flanco }\end{array}$ \\
\hline $\begin{array}{l}\text { Chu PL, Wei YF, } \\
\text { Huang JW, Chen } \\
\text { SI, Chu TS, Wu } \\
\text { KD }\end{array}$ & $\begin{array}{l}\text { Clinical } \\
\text { characteristics } \\
\text { of patients with } \\
\text { segmental renal } \\
\text { infarction }\end{array}$ & 2006 & Nephrology & $\begin{array}{l}22 \text { casos incluídos, } 16 \text { presentaron } \\
\text { dolor en flanco y dolor abdominal y } \\
22 \text { LDH elevada }\end{array}$ \\
\hline $\begin{array}{l}\text { Tsai SH, Chu SJ, } \\
\text { Chen SJ, Fan } \\
\text { YM, Chang WC, } \\
\text { Wu CP et al }\end{array}$ & $\begin{array}{l}\text { Acute renal } \\
\text { infarction: } \\
\text { a 10-year } \\
\text { experience }\end{array}$ & 2007 & $\begin{array}{l}\text { International } \\
\text { journal of } \\
\text { clinical } \\
\text { practice }\end{array}$ & $\begin{array}{l}18 \text { casos incluídos, } 13 \text { presentaron } \\
\text { dolor en flanco, } 13 \text { tenían glóbulos } \\
\text { blancos elevados, } 5 \text { PCR elevada y } \\
3 \text { LDH elevada }\end{array}$ \\
\hline $\begin{array}{l}\text { Huang CC, Chen } \\
\text { WL, Chen JH, } \\
\text { Wu YL, Shiao } \\
\text { CJ }\end{array}$ & $\begin{array}{l}\text { Clinical } \\
\text { Characteristics } \\
\text { of Renal } \\
\text { Infarction } \\
\text { in an Asian } \\
\text { Population } \\
\text { Chien-Cheng } \\
\end{array}$ & 2008 & $\begin{array}{l}\text { Annals of the } \\
\text { Academy of } \\
\text { Medicine, } \\
\text { Singapore }\end{array}$ & $\begin{array}{l}38 \text { casos incluídos, } 22 \text { presentaron } \\
\text { dolor abdominal, } 19 \text { dolor en } \\
\text { flanco y } 35 \text { presentaron LDH } \\
\text { elevada }\end{array}$ \\
\hline $\begin{array}{l}\text { Antopolsky M, } \\
\text { Simanovsky N, } \\
\text { Stalnikowicz } \\
\text { R, Salameh S, } \\
\text { Hiller N }\end{array}$ & $\begin{array}{l}\text { Renal infarction } \\
\text { in the ED: } \\
10 \text {-year } \\
\text { experience and } \\
\text { review of the } \\
\text { literature }\end{array}$ & 2011 & $\begin{array}{l}\text { American } \\
\text { Journal of } \\
\text { Emergency } \\
\text { Medicine }\end{array}$ & $\begin{array}{l}38 \text { casos incluídos, } 23 \text { presentaron } \\
\text { dolor abdominal, } 21 \text { dolor en } \\
\text { flanco, } 29 \text { proteinuria, } 26 \text { con LDH } \\
\text { elevada y } 3 \text { presentaron IRA }\end{array}$ \\
\hline
\end{tabular}




\begin{tabular}{|c|c|c|c|c|}
\hline $\begin{array}{l}\text { Rhee H, Song } \\
\text { SH, Won Lee D, } \\
\text { Lee SB, Kwak IS, } \\
\text { Seong EY }\end{array}$ & $\begin{array}{l}\text { The } \\
\text { significance } \\
\text { of clinical } \\
\text { features in the } \\
\text { prognosis of } \\
\text { acute renal } \\
\text { infarction: } \\
\text { Single center } \\
\text { experience }\end{array}$ & 2012 & $\begin{array}{l}\text { Clinical and } \\
\text { Experimental } \\
\text { Nephrology }\end{array}$ & $\begin{array}{l}67 \text { casos incluídos, } 40 \text { presentaron } \\
\text { proteinuria y } 38 \text { hematuria y } 27 \\
\text { presentaron IRA }\end{array}$ \\
\hline $\begin{array}{l}\text { Bourgault M, } \\
\text { Grimbert P, } \\
\text { Verret C, Pourrat } \\
\text { J, Herody M, } \\
\text { Halimi JM, et al. }\end{array}$ & $\begin{array}{l}\text { Acute renal } \\
\text { infarction: A } \\
\text { case series }\end{array}$ & 2013 & $\begin{array}{l}\text { Clinical Journal } \\
\text { of the American } \\
\text { Society of } \\
\text { Nephrology }\end{array}$ & $\begin{array}{l}94 \text { casos incluídos, } 52 \text { presentaron } \\
\text { dolor abdominal, } 46 \text { dolor en } \\
\text { flanco, } 85 \text { presentaron LDH } \\
\text { elevado y } 38 \text { IRA }\end{array}$ \\
\hline $\begin{array}{l}\text { Bae EJ, Hwang } \\
\text { K, Jang HN, Kim } \\
\text { MJ, Jeon DH, } \\
\text { Kim HJ, et al. }\end{array}$ & $\begin{array}{l}\text { A retrospective } \\
\text { study of short- } \\
\text { and long-term } \\
\text { effects on renal } \\
\text { function after } \\
\text { acute renal } \\
\text { infarction }\end{array}$ & 2014 & Ren Fail & $\begin{array}{l}100 \text { casos incluídos, } 91 \\
\text { presentaron dolor abdominal y/o } \\
\text { dolor en flanco, } 96 \text { LDH elevada y } \\
30 \text { IRA }\end{array}$ \\
\hline $\begin{array}{l}\text { Karacabey S, } \\
\text { Hocagil H, Sanri } \\
\text { E, Hocagil A, } \\
\text { Cuneyt Ardic S, } \\
\text { Suman E, et al. }\end{array}$ & $\begin{array}{l}\text { No suspicion, } \\
\text { no disease! } \\
\text { renal infarction: } \\
\text { Case series }\end{array}$ & 2014 & $\begin{array}{l}\text { Urology } \\
\text { Journal }\end{array}$ & $\begin{array}{l}5 \text { casos incluídos, } 4 \text { presentaron } \\
\text { dolor en flanco, } 4 \text { glóbulos blancos } \\
\text { elevados y presentaron proteinuria }\end{array}$ \\
\hline $\begin{array}{l}\text { Lin WL, Seak } \\
\text { CJ, Wu JY, Weng } \\
\text { YM, Chen HC }\end{array}$ & $\begin{array}{l}\text { Risk Factors for } \\
\text { Development } \\
\text { of Chronic } \\
\text { Kidney Disease } \\
\text { following Renal } \\
\text { Infarction: } \\
\text { Retrospective } \\
\text { Evaluation of } \\
\text { Emergency } \\
\text { Room Patients } \\
\text { from a Single } \\
\text { Center }\end{array}$ & 2014 & PLOS ONE & $\begin{array}{l}40 \text { casos incluídos, } 34 \text { presentaron } \\
\text { dolor abdominal, } 17 \text { dolor en } \\
\text { flanco y } 19 \text { progresaron a ERC } \\
\text { terminal }\end{array}$ \\
\hline $\begin{array}{l}\text { Nagasawa T, } \\
\text { Matsuda K, } \\
\text { Takeuchi Y, } \\
\text { Fukami H }\end{array}$ & $\begin{array}{l}\text { A case series } \\
\text { of acute renal } \\
\text { infarction at a } \\
\text { single center in } \\
\text { Japan }\end{array}$ & 2015 & $\begin{array}{l}\text { Clinical and } \\
\text { Experimental } \\
\text { Nephrology }\end{array}$ & $\begin{array}{l}33 \text { casos incluídos, } 21 \text { presentaron } \\
\text { dolor en flanco, } 33 \text { PCR elevada, } \\
30 \text { LDH elevada y } 25 \text { presentaron } \\
\text { IRA }\end{array}$ \\
\hline $\begin{array}{l}\text { Ongun S, } \\
\text { Bozkurt O, Demir } \\
\text { O, Cimen S, } \\
\text { Aslan G }\end{array}$ & $\begin{array}{l}\text { Midterm renal } \\
\text { functions } \\
\text { following acute } \\
\text { renal infarction }\end{array}$ & 2015 & $\begin{array}{l}\text { Kaohsiung } \\
\text { Journal of } \\
\text { Medical } \\
\text { Sciences }\end{array}$ & $\begin{array}{l}23 \text { casos incluídos, } 16 \text { presentaron } \\
\text { dolor en flanco, } 13 \text { dolor } \\
\text { abdominal, } 20 \text { presentaron LDH } \\
\text { elevada y } 7 \text { IRA }\end{array}$ \\
\hline $\begin{array}{l}\text { Oh YK, Yang } \\
\text { CW, Kim Y, Kang } \\
\text { S, Park CW, Kim } \\
\text { YS, et al. }\end{array}$ & $\begin{array}{l}\text { Clinical } \\
\text { Characteristics } \\
\text { and Outcomes } \\
\text { of Renal } \\
\text { Infarction }\end{array}$ & 2016 & $\begin{array}{l}\text { American } \\
\text { Journal } \\
\text { of Kidney } \\
\text { Diseases }\end{array}$ & $\begin{array}{l}438 \text { casos incluídos, } 233 \\
\text { presentaron dolor abdominal, } 219 \\
\text { dolor en flanco, } 131 \text { presentaron } \\
\text { hematuria y } 88 \text { IRA }\end{array}$ \\
\hline $\begin{array}{l}\text { Yang J, Lee JY, } \\
\text { Na YJ, Lim SY, } \\
\text { Kim MG, Jo SK, } \\
\text { et al. }\end{array}$ & $\begin{array}{l}\text { Risk factors } \\
\text { and outcomes } \\
\text { of acute renal } \\
\text { infarction }\end{array}$ & 2016 & $\begin{array}{l}\text { Kidney } \\
\text { Research } \\
\text { and Clinical } \\
\text { Practice } \\
\end{array}$ & $\begin{array}{l}89 \text { casos incluídos, } 37 \text { presentaron } \\
\text { dolor abdominal, } 13 \text { dolor en } \\
\text { flanco, } 37 \text { hematuria y } 36 \\
\text { proteinuria. } 31 \text { presentaron IRA }\end{array}$ \\
\hline $\begin{array}{l}\text { Mesiano P, } \\
\text { Rollino C, } \\
\text { Beltrame } \\
\text { G, Ferro M, } \\
\text { Quattrocchio G, } \\
\text { Fenoglio R. } \\
\end{array}$ & $\begin{array}{l}\text { Acute renal } \\
\text { infarction: a } \\
\text { single center } \\
\text { experience }\end{array}$ & 2016 & $\begin{array}{l}\text { Journal of } \\
\text { Nephrology }\end{array}$ & $\begin{array}{l}18 \text { casos incluídos, } 13 \text { presentaron } \\
\text { dolor en flanco, } 16 \text { LDH elevada y } \\
2 \text { presentaron IRA }\end{array}$ \\
\hline $\begin{array}{l}\text { Kwon JH, On BJ, } \\
\text { Ha SO, Kim DY, } \\
\text { Do HH. }\end{array}$ & $\begin{array}{l}\text { Renal } \\
\text { Complications } \\
\text { in Patients } \\
\text { with Renal } \\
\text { Infarction: } \\
\text { Prevalence and } \\
\text { Risk Factors }\end{array}$ & 2016 & $\begin{array}{l}\text { Kidney and } \\
\text { Blood Pressure } \\
\text { Research }\end{array}$ & $\begin{array}{l}105 \text { casos incluídos, } 69 \\
\text { presentaron dolor abdominal, } 66 \\
\text { dolor en flanco, } 97 \text { LDH elevada y } \\
41 \text { presentaron IRA }\end{array}$ \\
\hline
\end{tabular}


Tabla 2: Artículos seleccionados. E

\begin{tabular}{|l|l|l|l|l|}
\hline $\begin{array}{l}\text { Caravaca-Fontán } \\
\text { F, Pampa Saico } \\
\text { S, Elías Triviño S, } \\
\text { Galeano Álvarez } \\
\text { C, Gomis Couto } \\
\text { A, Pecharromán } \\
\text { de las Heras I, } \\
\text { et al. }\end{array}$ & $\begin{array}{l}\text { Infarto renal } \\
\text { clínicas y } \\
\text { factores } \\
\text { pronósticos }\end{array}$ & 2016 & Nefrología & $\begin{array}{l}\text { 62 casos incluídos, 60 presentaron } \\
\text { dolor abdominal y 56 dolor en } \\
\text { flanco }\end{array}$ \\
\hline $\begin{array}{l}\text { Kagaya S, Yoshie } \\
\text { O, Fukami H, } \\
\text { Sato H, Saito } \\
\text { A, Takeuchi Y, } \\
\text { et al. }\end{array}$ & $\begin{array}{l}\text { Renal infarct } \\
\text { volume and } \\
\text { renal function } \\
\text { decline in acute } \\
\text { and chronic } \\
\text { phases }\end{array}$ & 2017 & $\begin{array}{l}\text { Clinical and } \\
\text { Experimental } \\
\text { Nephrology }\end{array}$ & $\begin{array}{l}39 \text { casos incluídos, 26 presentaron } \\
\text { dolor en flanco y/o abdominal }\end{array}$ \\
\hline
\end{tabular}

\section{B) Características clínicas y paraclínicas}

Con respecto a la edad media de las series fue de $60.1 \pm 15.7$ años (564 pacientes). Un 59\% (784/1330) de los pacientes de las series eran del sexo masculino.

En la tabla 3 se presentan las principales características clínicas halladas y en la tabla 4 las alteraciones de las variables paraclínicas analizadas.

\begin{tabular}{|l|l|}
\hline \multicolumn{2}{|c|}{ Características clínicas } \\
\hline Oliguria & $62.9 \%(83 / 132)$ \\
\hline Dolor en flanco & $56.4 \%(709 / 1257)$ \\
\hline Dolor abdominal & $48.4 \%(574 / 1186)$ \\
\hline Náuseas & $25.8 \%(13 / 1215)$ \\
\hline Fiebre & $16.6 \%(203 / 1224)$ \\
\hline Vómitos & $14 \%(152 / 1087)$ \\
\hline Disnea & $6.6 \%(34 / 516)$ \\
\hline Dolor torácico & $4.8 \%(23 / 476)$ \\
\hline Confusión & $4 \%(20 / 483)$ \\
\hline Diarrea & $4 \%(19 / 476)$ \\
\hline
\end{tabular}

\begin{tabular}{|l|l|}
\hline \multicolumn{2}{|c|}{ Características paraclínicas } \\
\hline LDH alterada & $91.6 \%(482 / 526)$ \\
\hline PCR alterada & $82.1 \%(124 / 151)$ \\
\hline GB alterada & $69.2 \%(202 / 292)$ \\
\hline Presencia de hematuria & $41.8 \%(472 / 1128)$ \\
\hline ALT alterada & $40.4 \%(44 / 109)$ \\
\hline Presencia de proteinuria & $32.4 \%(299 / 922)$ \\
\hline Creatinina alterada & $26.8 \%(46 / 168)$ \\
\hline
\end{tabular}

\section{C) Retraso diagnóstico e impacto en la función renal}

De acuerdo a la información revisada hay un período de $4.8 \pm 5.6$ días entre el inicio de síntomas al diagnóstico (Bourgault (27), Tsai ${ }^{(28)}$ expresan período de tiempo como medias $\mathrm{n}=$ 112). Desde la consulta en el centro médico al diagnóstico hay un período de $3.3 \pm 1.3$ días (Korzets ${ }^{(29)}$, Lumerman ${ }^{(30)}$, Oh ${ }^{(31)}$ expresan período de tiempo en medias $n=40$ ).

Con respecto al impacto en la función renal, encontramos que se identificó la injuria renal aguda, según la clasificación RIFLE en las series de Yang ${ }^{(32)}$, Nagasawa ${ }^{(33)}$ y Rhee (26), la clasificación AKIN fue utilizada por Mesiano ${ }^{(34)}$ y la clasificación KDIGO por Bae ${ }^{(4)}$, Oh ${ }^{(3)}$ y Kwon (35). En la serie de Ongun (36) y Domanovits ${ }^{(37)}$ se define por un aumento de la creatinina por encima de 1,3 mg/dl. En las series de Lessman ${ }^{(38)}$, Karacabey ${ }^{(39)}$, Korzets ${ }^{(29)}$, Iga ${ }^{(40)}$ y Winzelberg (41) no se aclara como se identificaron los pacientes con injuria renal aguda. 
En las series de Caravaca-Fontan (42), Yang (32), Lessman (38), Ongun (36), Oh ${ }^{(3)}$, Kwon (35), Lin (43) la enfermedad crónica se definió por el $\mathrm{FG}<60 \mathrm{ml} / \mathrm{min}$ por lo menos por 3 meses. En la serie de $\mathrm{Bae}^{(4)}$ se define por la persistencia de las alteraciones definidas por la clasificación KDIGO por al mes 3 meses. Las series de $\mathrm{Chu}^{\left({ }^{44)}\right.}$ y Huang ${ }^{(45)}$ la definen por la persistencia de crea mayor a $1,5 \mathrm{mg} / \mathrm{dl}$ por meses. Y en el caso de Domanovits ${ }^{(37)}$ por el aumento de Crea por encima de $1,3 \mathrm{mg} / \mathrm{dl}$ por meses. Las series de Karacabey ${ }^{(39)}$, Bourgault ${ }^{(27)}$, Tsai ${ }^{(28)}$ y Korzets ${ }^{(29)}$ no aclaran como la definen.

En la tabla 5 se presentan el impacto de la función renal encontrada en estos pacientes.

\begin{tabular}{|l|l|}
\hline \multicolumn{2}{|c|}{ Impacto en la función renal } \\
\hline Injuria renal aguda & $30.8 \%(327 / 1061)$ \\
\hline Enfermedad renal crónica & $17.3 \%(152 / 881)$ \\
\hline Enfermedad renal terminal & $5.2 \%(43 / 834)$ \\
\hline
\end{tabular}

\section{Discusión}

Los síntomas y signos que se repiten en la mayoría de las series y que deberían hacer sospechar el diagnostico son: dolor en flanco y/o abdominal, nauseas, vómitos, fiebre y oliguria. La oliguria que es un dato registrado en pocas series pero que constituye el signo clínico que aparece con mayor frecuencia en nuestra revisión $(62.9 \%$ vs 56.45 y $48 \%$ del dolor en flanco y dolor abdominal respectivamente).

Los 3 parámetros serológicos que se encuentran alterados en mayor proporción son: LDH, PCR y leucocitosis.

Se ha asociado al pico del valor de la LDH con el volumen del infarto y con la disminución del filtrado glomerular en la fase aguda del infarto pero no en la fase crónica ${ }^{(46)}$. Esto haría a la LDH como la opción más apropiada dentro de los marcadores serológicos para despistar el diagnostico en vista a solicitar una tomografía con contraste o si se tuviera una tomografía no concluyente como un elemento para orientar el diagnostico.

Es necesario valorar cuales son los síntomas más frecuentes en el infarto renal e insistir en el diagnóstico temprano dado que el tratamiento endovascular (trombólisis, trombectomía y colocación de stent) solo se pueden realizar en las primeras horas de la consulta (3-24 horas y se destaca que aún son experiencias aisladas)(47).

Para disminuir esta brecha diagnóstica de días a horas (se observó una media de 3 días de arribo a emergencia al diagnóstico), es necesaria una alta sospecha clínica. De los antecedentes personales, hay que destacar que la cardioembolia en general y la fibrilación auricular en particular son la principal causa de infarto renal. En las series estudiadas, el 41,8\% (514/1231) de los casos presentaban fibrilación auricular.

El impacto a nivel renal del infarto puede llevar a la falla renal. En el trabajo actual se constató injuria renal aguda en $30.8 \%$ (327/1061) de los pacientes, la progresión a enfermedad renal crónica se observó en $17.3 \%$ (152/881) de los pacientes y la progresión a enfermedad renal terminal se observó en $5.2 \%$ (43/834) de los pacientes. Estos valores son más elevados que los valores observados en la serie más grande de Oh et al. (3)

Se destaca que la injuria renal aguda es multifactorial. Debido a la condición de fuente de información secundaria, no fue posible asociar variables con el desarrollo de injuria renal aguda y enfermedad renal crónica en el trabajo actual.

Previamente se ha reportado que el desarrollo de injuria renal tanto aguda como progresión a la enfermedad renal crónica se asocia a mayor edad, diabetes mellitus ${ }^{(32)}$, y mayor volumen del infarto ${ }^{(46)}$. Y se ha relacionado la ocurrencia de injuria renal con el grado de proteinuria y la presencia de hematuria microscópica, niveles más altos de AST, ALT, LDH (4) y PCR ${ }^{(32)}$.

Dado lo explicado en párrafos anteriores se debería controlar de forma más estricta la función renal de pacientes con infarto renal de edad más avanzada, con diabetes, con mayor área infartada y con LDH y PCR elevadas.

La primera limitación de nuestro trabajo dada su condición de revisión, es el sesgo de publicación. En segundo lugar hay variables que podrían influir en la repercusión de la función renal y que no pudieron ser evaluadas dado que este trabajo es una fuente de información secundaria y por lo tanto se limita a los datos que fueron estudiados en los trabajos originales. 
Y en tercer lugar, la heterogeneidad en la forma de determinar la injuria renal entre los diferentes estudios podría influir en los resultados de nuestro trabajo.

\section{Conclusiones}

El infarto renal es una entidad poco frecuente y sub-diagnosticada que debería sospecharse frente a pacientes con antecedente personal de patologías que predispongan a la cardioembolia (como la fibrilación auricular) y que se presenten con dolor en flanco y/o abdominal y LDH aumentado. La sospecha clínica puede llevar a un diagnóstico oportuno que habilita la realización de terapias endovasculares. En la series de casos estudiados alrededor de 1 de cada 3 pacientes con infarto renal desarrollaran injuria renal aguda y alrededor de 1 de cada 6 progresara a enfermedad renal crónica.

\section{Conflicto de interés}

No existe conflicto de interés.

\section{Bibliografía}

1- Paris B, Bobrie G, Rossignol P, Le Coz S, Chedid A, Plouin PF. Blood pressure and renal outcomes in patients with kidney infarction and hypertension J Hypertens. 2006; 24(8):1649-54.

2- Hoxie J, Coggin C. Renal infarction statistical study of two hundred and five cases and detailed report of an unusual case. Arch Intern Med (Chic). 1940;65(3):587-594

3- Oh YK, Yang CW, Kim Y, Kang S, Park CW, Kim YS, et al. Clinical Characteristics and Outcomes of Renal Infarction. Am J Kidney Dis. 2016; 67 (2):243-50.

4- Bae EJ, Hwang K, Jang HN, Kim MJ, Jeon D, Kim H, et al. A retrospective study of short- and longterm effects on renal function after acute renal infarction. Ren Fail. 2014;36 (9):1385-9.

5- Bellomo R, Ronco C, Kellum JA, Mehta RL, Palevsky P; Acute Dialysis Quality Initiative workgroup. Acute renal failure-definition, outcome measures, animal models, fluid therapy and information technology needs: the Second International Consensus Conference of the Acute Dialysis Quality Initiative (ADQI) Group. Crit Care. 2004;8(4):R204-12.

6- Mehta RL, Kellum JA, Shah SV, Molitoris BA, Ronco C, Warnock DG, et al. Acute Kidney Injury Network: report of an initiative to improve outcomes in acute kidney injury. Crit Care. 2007;11:R31.

7- Kidney Disease: Improving Global Outcomes (KDIGO). Acute Kidney Injury Work Group. KDIGO clinical practice guidelines for acute kidney injury. Kidney Int Suppl. 2012;2:2.

8- Levey A, Coresh J. Chronic kidney disease. Lancet. 2011;379:165-80.

9- Regan FC, Crabtree EG. Renal Infarction: A Clinical and Possible Surgical Entity 1. J Urol. 1948;59(6):981-1014.

10- Heitzman ER, Perchik L. Radiographic features of renal infarction; review of 13 cases. Radiology. 1961;76(June):39-46.

11- Hazanov N, Somin M, Attali M, Beilinson N, Thaler M, Mouallem M, et al. Acute renal embolism: Fortyfour cases of renal infarction in patients with atrial fibrillation. Medicine (Baltimore). 2004;83(5):292-9.

12- Krämer SC, Seifarth H, Pamler R, Fleiter T, Bühring J, Sunder-Plassmann L, et al. Renal infarction following endovascular aortic aneurysm repair: incidence and clinical consequences. J Endovasc Ther. 2002;9(1):98-102.

13- Böckler D, Krauss M, Mannsmann U, Halawa M, Lange R, Probst T, et al. Incidence of Renal Infarctions after Endovascular AAA Repair: Relationship to Infrarenal versus Suprarenal Fixation. J Endovasc Ther. 2003 Dec;10(6):1054-60.

14- Janower M, Weber A. Radiologic evaluation of acute renal infarction. Am J Roentgenol Radium Ther Nucl Med. 1965;95(2):309-17.

15- Lang EK. Arteriographic diagnosis of renal infarcts. Radiology. 1967;88(6):1110-6.

16- Lang EK, Mertz JHO, Nourse M. Renal Arteriography in the Assessment of Renal Infarction. J Urol. 1968; 99(5):506-12.

17- Suzer O, Shirkhoda A, Jafri SZ, Madrazo BL, Bis KG, Mastromatteo JF. CT features of renal infarction. Eur J Radiol. 2002; 44(1):59-64. 
18- Romano S, Scaglione M, Gatta G, Lombardo P, Stavolo C, Romano L, et al. Association of splenic and renal infarctions in acute abdominal emergencies. Eur J Radiol. 2004; 50(1):48-58.

19- Huang CC, Lo HC, Huang HH, Kao WF, Yen DH, Wang LM, et al. ED presentations of acute renal infarction. Am J Emerg Med. 2007; 25(2):164-9.

20- Wong W, Moss A, Federle M, Cochran S, London S. Renal infarction: CT diagnosis and correlation between CT findings and etiologies. Radiology. 1984;150: 201-5.

21- Bertolotto M, Martegani A, Aiani L, Zappetti R, Cernic S, Cova MA. Value of contrast-enhanced ultrasonography for detecting renal infarcts proven by contrast enhanced CT. A feasibility study. Eur Radiol. 2008;18(2):376-83.

22- Faucon A-L, Bobrie G, Jannot A-S, Azarine A, Plouin P-F, Azizi M, et al. Cause of renal infarction: a retrospective analysis of 186 consecutive cases. J Hypertens. 2018; 36(3):634-640

23- Yoon K, Song SY, Lee CH, Ko BH, Lee S, Kang BK, et al. Spontaneous Renal Artery Dissection as a Cause of Acute Renal Infarction: Clinical and MDCT Findings. J Korean Med Sci. 2017;32:605-12.

24- Hilton S, Bosniak MA, Raghavendra BN, Subramanyam BR, Rothberg M, Megibow AJ. CT findings in acute renal infarction. Urol Radiol. 1984;6(3-4):158-63.

25- Bolderman R, Oyen R, Verrijcken A, Knockaert D, Vanderschueren S. Idiopathic renal infarction. Am J Med. 2006;119 (4):9-12.

26- Rhee H, Song SH, Won Lee D, Lee SB, Kwak IS, Seong EY. The significance of clinical features in the prognosis of acute renal infarction: Single center experience. Clin Exp Nephrol. 2012;16(4):611-6.

27- Bourgault M, Grimbert P, Verret C, Pourrat J, Herody M, Halimi JM, et al. Article Acute Renal Infarction : A Case Series. Clin J Am Soc Nephrol. 2013 Mar;8(3):392-8.

28- Tsai S-H, Chu S-J, Chen S-J, Fan Y-M, Chang W-C, Wu C-P, et al. Acute renal infarction: a 10-year experience. Int J Clin Pract. 2007 Jan;61(1):62-7.

29- Korzets Z, Plotkin E, Bernheim J, Zissin R. The clinical spectrum of acute renal infarction. Isr Med Assoc J. 2002;4(10):781-4

30- Lumerman JH, Hom D, Eiley D, Smith AD. Heightened Suspicion and Rapid Evaluation with CT for Early Diagnosis of Partial Renal Infarction. J Endourol. 1999;13(3):209-14.

31- Oh YK. The association between acute kidney injury in renal infarction and progression to chronic kidney disease. Kidney Res Clin Pract. 2016; 35 (3):192-3.

32- Yang J, Lee JY, Na YJ, Lim SY, Kim MG, Jo SK, et al. Risk factors and outcomes of acute renal infarction. Kidney Res Clin Pract. 2016;35(2):90-5.

33- Nagasawa T, Matsuda K, Takeuchi Y, Fukami H. A case series of acute renal infarction at a single center in Japan. Clin Exp Nephrol. 2016 Jun;20 (3):411-5.

34- Mesiano P, Rollino C, Beltrame G, Ferro M, Quattrocchio G, Fenoglio R, et al. Acute renal infarction: a single center experience. J Nephrol. 2017;30 (1):103-7.

35- Kwon JH, Oh BJ, Ha SO, Kim DY, Do HH. Renal Complications in Patients with Renal Infarction Prevalence and Risk Factors. Kidney Blood Press Res. 2016;41(6):865-72.

36- Ongun S, Bozkurt O, Demir O, Cimen S, Aslan G. Midterm renal functions following acute renal infarction. Kaohsiung J Med Sci. 2015;31(10):529-33.

37- Domanovits H, Paulis M, Nikfardjam M, Meron G, Kurkciyan I, Bankier A, et al. Acute renal infarction. Clinical characteristics of 17 patients. Medicine (Baltimore). 1999;78:386-94.

38- Lessman R, Johnson S, Courn J, Kaufman J. Renal Artery Embolism. Clinical Features and Long-Term Follow-up of 17 Cases. Ann Intern Med. 1978;89(4):477-82.

39- Karacabey S, Hocagil H, Sanri E, Hocagil AC, Ardic S, Suman E. No suspicion, no disease! renal infarction: Case series. Urol J. 2014;11(6):1984-6.

40- Iga K, Izumi C, Nakano A, Sakanoue Y, Kitaguchi S, Himura Y, et al. Problems in the initial diagnosis of renal infarction. Intern Med. 1997;36(5):330-2.

41- Winzelberg GG, Hull JD, Agar JWM, Rose BD, Pletka PG. Elevation of Serum Lactate Dehydrogenase Levels in Renal Infarction. JAMA. 1979;242:268-9. 
42- Caravaca-Fontán F, Pampa Saico S, Elías Triviño S, Galeano Álvarez C, Gomis Couto A, Pecharromán de las Heras I, et al. Infarto renal agudo: características clínicas y factores pronósticos. Nefrología (Madrid). 2016;36(2):141-8.

43- Lin W-L, Seak C-J, Wu J-Y, Weng Y-M, Chen H-C. Risk Factors for Development of Chronic Kidney Disease following Renal Infarction: Retrospective Evaluation of Emergency Room Patients from a Single Center. PLoS One. 2014;9 (6):e98880.

44- Chu P-L, Wei Y-F, Huang J-W, Chen S-I, Chu T-S, Wu K-D. Clinical characteristics of patients with segmental renal infarction. Nephrology. 2006;11(4):336-40.

45- 4Huang C-C, Chen W-L, Chen J-H, Wu Y-L, Shiao C-J. Clinical characteristics of renal infarction in an Asian population. Ann Acad Med Singapore. 2008;37(5):416-20.

46- Kagaya S, Yoshie O, Fukami H, Sato H, Saito A, Takeuchi Y, et al. Renal infarct volume and renal function decline in acute and chronic phases. Clin Exp Nephrol. 2017;21(6):1030-4.

47- Piffaretti G, Riva F, Tozzi M, Lomazzi C, Rivolta N, Carrafiello G, et al. Vascular and Endovascular Surgery Catheter-Directed Thrombolysis for Acute Renal Artery Thrombosis : Report of 4 Cases. Vasc Endovascular Surg. 2008 Aug-Sep;42(4):375-9.

\section{Aporte cada autor al trabajo}

Ana Carina Pizzarossa: concepción del trabajo, búsqueda de artículos y datos. Procesamiento de datos. Análisis de resultados. Redacción de discusión.

Marina Rodríguez: Búsqueda de artículos y datos. Procesamiento de datos. Corrección del artículo. 